Check for updates

Cite this: RSC Adv., 2019, 9, 24057

\title{
Solvent-controlled synthesis of multicolor photoluminescent carbon dots for bioimaging $\dagger$
}

Received 5th June 2019 Accepted 12th July 2019

DOI: $10.1039 / \mathrm{c} 9 \mathrm{ra0} 4241 \mathrm{e}$

rsc.li/rsc-advances

\begin{abstract}
Yang Yan, (D) $t^{\mathrm{ab}}$ Longyu Xia (D) $t^{\mathrm{ab}}$ and Lan Ma (D) *b
Multicolor fluorescent carbon dots (CDs) have potential applications in multichannel detection and multicolor imaging. In this study, multicolor fluorescent CDs were synthesized by changing the solvent type and adjusting the reactant ratio. The four prepared CDs emitted bright and stable blue (B-), green $(G-)$, yellow $(Y-)$, and red (R-) fluorescence under a single UV light ( $\lambda_{\text {ex }}=365 \mathrm{~nm}$ ). The photoluminescence (PL) emission wavelengths changed from $445 \mathrm{~nm}$ for B-CDs to $620 \mathrm{~nm}$ for R-CDs, and therefore covered the entire visible spectrum. The absolute quantum yields for the $\mathrm{B}_{-}, \mathrm{G}_{-}, \mathrm{Y}_{-}$, and R-CDs were $27.3 \%, 31.1 \%, 22.9 \%$, and $8.8 \%$, respectively. Characterization of the CDs showed that the differences among the optical features of the four prepared CDs arise from the differences among the surface states and nitrogen-derived structures in the carbon core. The four prepared CDs all showed low toxicity and steady $\mathrm{PL}$, and therefore have potential applications in both in vitro and in vivo imaging
\end{abstract}

\section{Introduction}

Carbon dots (CDs) are carbon-based zero-dimensional materials, typically of size less than $10 \mathrm{~nm}$. They were discovered by $\mathrm{Xu}$ et al. at the University of South Carolina, during separation of single-walled carbon nanotubes. ${ }^{\mathbf{1}}$ Because of their properties such as low cost, low toxicity, an adjustable luminescence range, excellent light stability, and absence of photobleaching and light flickering, CDs have a broad range of potential applications, e.g., in optical biomarkers, ${ }^{2-4}$ drug delivery, ${ }^{4,5}$ cancer diagnosis, ${ }^{6}$ chemical sensing, ${ }^{7-10}$ catalysis, ${ }^{\mathbf{1 1}}$ light-emitting devices, ${ }^{\mathbf{1 2}}$ and bioimaging, ${ }^{13}$ and have attracted wide research interest.

There are two main methods for the synthesis of CDs, i.e., the top-down method and the bottom-up method. In the top-down method, a large carbon skeleton, e.g., graphite, ${ }^{\mathbf{1 4}}$ carbon nanotubes, ${ }^{15}$ carbon fibers, ${ }^{\mathbf{1 6 , 1 7}}$ carbon black, ${ }^{\mathbf{1 8}}$ fullerene, ${ }^{19}$ coal pitch, ${ }^{20}$ and candle ash, ${ }^{21}$ are directly

\footnotetext{
${ }^{a}$ Department of Chemistry, Tsinghua University, Beijing 100084, P. R. China

${ }^{b}$ Division of Life Science and Health, Tsinghua University Graduate School at Shenzhen, Shenzhen 518055, P. R. China. E-mail: malan@sz.tsinghua.edu.cn; Tel: +86-0755-26033033

$\dagger$ Electronic supplementary information (ESI) available: Summary of PL lifetimes of the four as-prepared CDs. PL QY data of the four as-prepared CDs under their optimal excitation wavelengths. The X-ray diffraction (XRD) patterns of the four as-prepared CDs. The XPS full survey spectrum of four as-prepared CDs. Elemental analysis results of the four as-prepared CDs. XPS data analysis of the four as-prepared CDs. The PL emission of the four as-prepared CDs dispersed in water. The impact of ion strength to the PL emission of the four as-prepared CDs. The impact of solvent content to the PL emission of the four as-prepared CDs. Viability of MDA-MB-231 cells after $24 \mathrm{~h}$ incubation with different concentration of all the four as-prepared CDs. See DOI: 10.1039/c9ra04241e $\$$ The two authors contributed equally.
}

stripped to smaller carbon materials via treatments such as chemical oxidation, ${ }^{\mathbf{1 6}}$ laser etching, ${ }^{\mathbf{1 8 , 2 2}}$ and electrochemical methods. ${ }^{23-25}$ These usually require expensive equipment and are time consuming. Moreover, the size and properties of the CDs cannot be effectively regulated. The bottom-up method is relatively simple and easy to control. CDs are generated from small carbon particles or small molecules by various methods, e.g., microwave-assisted, ${ }^{6,26,27}$ hydrothermal, ${ }^{2,3,9,12,28-47}$ combustion, ${ }^{21}$ and templating methods. ${ }^{48}$ This is therefore the prevalent approach.

Multicolor fluorescent CDs play a vital role in multichannel detection and multicolor imaging. Much work has been done on the fabrication of multicolor CDs. Ding et al. obtained multicolor CDs via a time-consuming silica-gel column separation process. ${ }^{49}$ Bao et al. synthesized three types of fluorescent CDs by wet oxidation of carbon fibers; the method involved the use of a strong acid and the quantum yields (QYs) were low. ${ }^{16}$ Ding et al. and Zhan et al. synthesized multicolor fluorescent CDs by using different solvents. ${ }^{50,51}$ The solvothermal method is simple and the fluorescence QYs are good. However, most of the raw materials are toxic, harmful, and environmentally unfriendly, and are potentially hazardous in practical applications. From this perspective, non-toxic citric acid (CA) and urea are excellent precursors for CDs fabrication. $\mathrm{CA}$ and urea have long been used as the raw materials for CDs synthesis but there are few reports of their use in the synthesis of red fluorescent CDs. In this study, we used simple, inexpensive, green, non-toxic CA and urea as the raw materials and obtained blue, green, yellow, and red fluorescent CDs (B-, G-, Y-, and R-CDs) simply by changing the solvent and the ratio of the raw materials. The 
morphologies, sizes, compositions, and optical properties of the prepared CDs were investigated. The results show that the maximum emission wavelength of the CDs is affected by the surface states and content of pyridinic N. A high pyridinic $\mathrm{N}$ content promotes redshifts of the maximum emission wavelength and the surface states play an important role in the CDs properties. Because of the low toxicity, good biocompatibility, and stability of the prepared CDs, they can be used in both in vitro and in vivo imaging.

\section{Experimental section}

\subsection{Materials}

All chemicals were commercially available, of analytical grade, and used without further purification. CA, urea, ethanol, formic acid, ethyl acetate, sodium chloride, potassium chloride, sodium dihydrogen phosphate, and monopotassium phosphate were purchased from the Macklin Biochemical Co., Ltd. (Shanghai, China). Milli-Q water (resistivity 18.8 $\mathrm{M} \Omega$ ) was used in all experiments. Roswell Park Memorial Institute-1640 medium (PRMI-1640) was purchased from GIBCO (Thermo, USA) and fetal bovine serum (FBS) was purchased from ExCell Bio (Shanghai, China). Cell Counting Kit-8 (CCK-8) solution was purchased from Yeasen (Shanghai, China).

\subsection{Synthesis of multicolor CDs}

CA $(1.000 \mathrm{~g})$ and urea $(2.000 \mathrm{~g})$ were added to $0.01 \mathrm{M} \mathrm{HCl}(10$ $\mathrm{mL}$, B-CDs), ethanol (10 mL, G-CDs), or formic acid $(10 \mathrm{~mL}$, R-CDs). The mixture was transferred to a $30 \mathrm{~mL}$ Teflon-lined stainless-steel autoclave. The sealed autoclave was heated to $160{ }^{\circ} \mathrm{C}$ in an electric oven and then kept for $4 \mathrm{~h}$. For B- and G$\mathrm{CDs}$, the mixture was cooled to room temperature, centrifuged at $16000 \mathrm{rpm}$ for $10 \mathrm{~min}$ to remove large particles, and then dialyzed with a molecular-weight cut-off (MWCO: 1000) membrane for $24 \mathrm{~h}$. The solution was freeze-dried for further characterization. For R-CDs, the solution was washed several times with ethyl acetate until solid arise. For Y-CDs synthesis, CA (1.500 g) and urea (1.500 g) were used; the procedure was otherwise the same as that for R-CDs.

\subsection{Cell culture}

MDA-MB-231, human breast cancer cells, were used in the bioimaging experiments. They were cultured in PRMI-1640 medium containing $10 \% \mathrm{FBS}$ at $37{ }^{\circ} \mathrm{C}$ in $5 \% \mathrm{CO}_{2}$.

\subsection{Cell toxicity assay}

MDA-MB-231 cells were used to evaluate the cytotoxicity of the B-CDs, G-CDs, Y-CDs, and R-CDs. A suspension $(100 \mu \mathrm{L})$ of the cells in the exponential growth phase was seeded into each well of a 96-well plate at a density of $5 \times 10^{3}$ cells per well. After culturing for $24 \mathrm{~h}$, CDs at different concentrations were cultured with the cells for another $24 \mathrm{~h}$. Cells cultured without CDs were used as a control. After incubation, the cells were washed with phosphate-buffered saline (PBS, $0.01 \mathrm{M}, \mathrm{pH}$ 7.4). PRMI-1640 medium $(100 \mu \mathrm{L})$ containing
$10 \%$ CCK- 8 was added to each well and were incubated at $37{ }^{\circ} \mathrm{C}$ for $1.5 \mathrm{~h}$ until the solvent turned yellow. The optical density at $450 \mathrm{~nm}$ of the mixture in each well was recorded with a Thermo Scientific Varioskan Flash Multimode reader. To accurately determine the viability of each test group, six replicate wells were used. The results are reported as the mean \pm the standard deviation. The cell viability was calculated as

$$
\begin{aligned}
\text { Cell viability } & =\left(\mathrm{OD}_{\text {samples }}-\mathrm{OD}_{\text {blank }}\right) /\left(\mathrm{OD}_{\text {control }}-\mathrm{OD}_{\text {blank }}\right) \\
& \times 100 \% .
\end{aligned}
$$

\subsection{Cell imaging}

MDA-MB-231 cells in the exponential phase were seeded into 24-well plates with aseptic coverslips at a concentration of $2.5 \times$ $10^{4}$ cells per well. These cells were cultured at $37^{\circ} \mathrm{C}$ for $24 \mathrm{~h}$, with $5 \% \mathrm{CO}_{2}$ for cell attachment. A CDs solution $\left(50 \mu \mathrm{g} \mathrm{mL} \mathrm{m}^{-1}\right.$ in the culture medium) was added to the MDA-MB-231 cells to replace the original medium. After growing for $24 \mathrm{~h}$, PBS was used to wash away the CDs solution. The cells were fixed by adding $4 \%$ paraformaldehyde $(500 \mu \mathrm{L})$ at room temperature. After $15 \mathrm{~min}$, PBS was added to the well to wash away the paraformaldehyde. The samples were then ready for imaging. The fluorescence was visualized with a Nikon A1R+ confocal laser scanning microscope equipped with a spectral detection system.

\subsection{Quantum yield measurements}

The QYs of the CDs were determined by using an integrating sphere attached to a Hamamatsu Quantaurus instrument. For example, ethanol solution of the B-CDs was diluted to an optical absorption below 0.1 at an excitation wavelength of $371 \mathrm{~nm}$. The ethanol solution was added to a $1 \mathrm{~cm}$ fluorescence cuvette, which was placed in the integrating sphere, and excitation was performed with $371 \mathrm{~nm}$ monochromatic light. PL spectra were recorded in the range $400-600 \mathrm{~nm}$. Fluorescence software was used to calculate the QY of the B-CDs from the PL spectrum. Each experiment was repeated three times in parallel and the average QY of the B-CDs was obtained. The QYs of all the samples were determined by using the above procedures and corresponding conditions.

\subsection{Characterization}

Transmission electron microscopy (TEM) and high-resolution TEM (HRTEM) images were obtained with a Tecnai G2 F30 instrument (FEI Company, USA) at $300 \mathrm{kV}$ to investigate the sizes and morphologies of the CDs samples. Atomic force microscopy (AFM; Bruker Innova, Germany) images were captured with a multimode in tapping mode. X-ray diffraction (XRD) patterns were recorded with an X-ray diffractometer (Bruker D8 Advance, Germany) with $\mathrm{Cu} \mathrm{K} \alpha$ radiation $(\lambda=$ $0.15418 \mathrm{~nm}$ ). Raman spectra were recorded with a LabRAM HR800 instrument (HORIBA, USA) with a $785 \mathrm{~nm}$ laser beam. Absorption spectra were recorded with a UV-vis 
spectrophotometer (Amersham Biosciences, USA). Fluorescence spectra were recorded with a luminescence spectrometer (Thermo Scientific Lumina, USA) with a slit width of $5 \mathrm{~nm}$. Fluorescence lifetimes were determined with a Fluorolog TCSPC instrument (HORIBA Scientific, Kyoto, Japan). Fouriertransform infrared (FT-IR) spectra were recorded (KBr pellets) with a Nicolet iS50 instrument (Thermo Fisher Scientific, USA) from 4000 to $500 \mathrm{~cm}^{-1}$. X-ray photoelectron spectroscopy (XPS) was performed with a PHI 5000 Versa Probe II spectrometer (Ulvac-Phi, Japan). Mice were imaged with a Bruker imaging system (Bruker In-Vivo F PRO, Germany). This study was performed in strict accordance with requirement of Experiments Animal Ethics and Welfare guideline of China and was approved by the Institutional Animal Care and Use Committee of Tsinghua University Graduate School at Shenzhen, Shenzhen, People's republic of China.

(a)

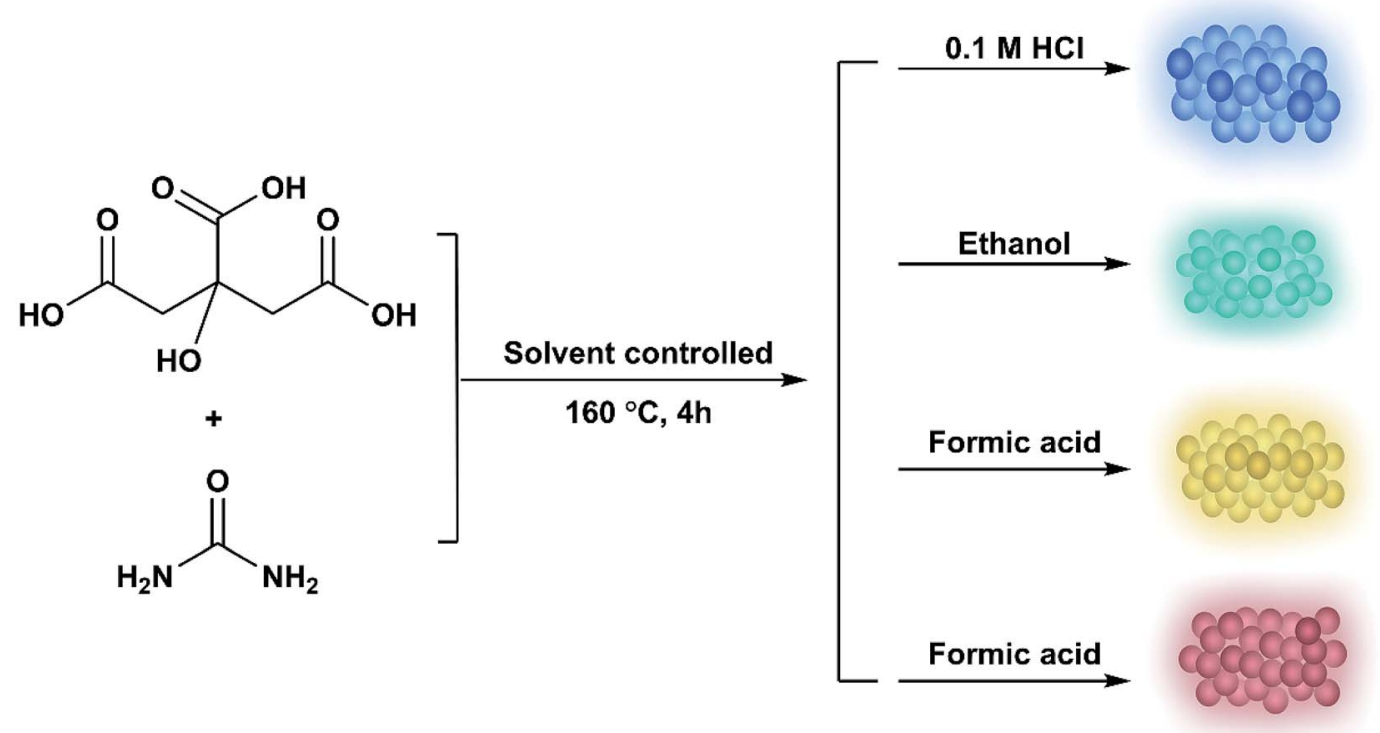

(b)

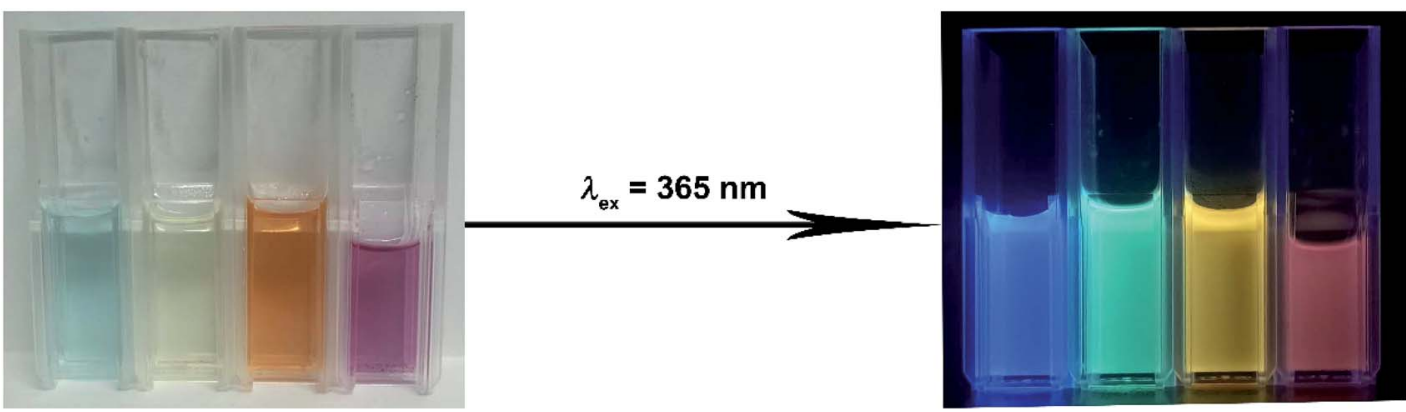

(c)

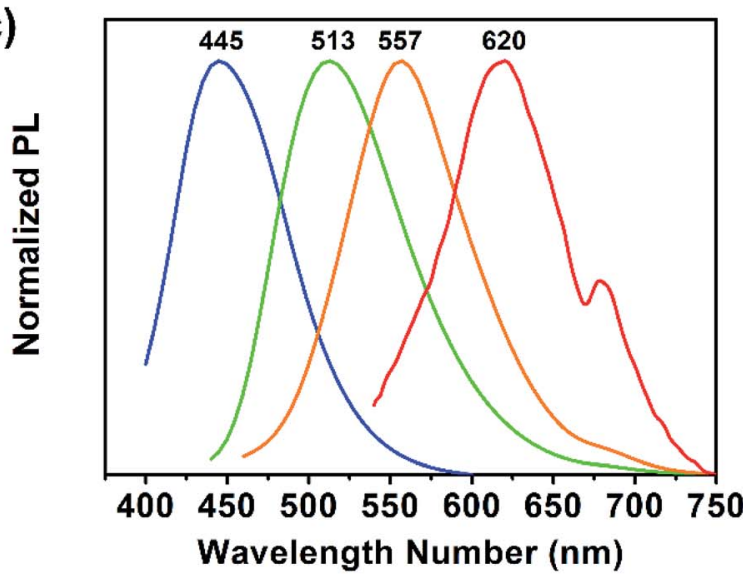

(d)

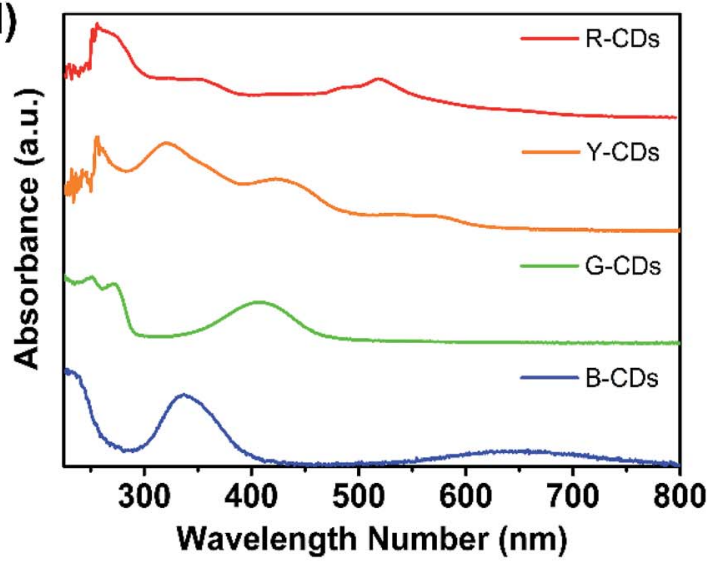

Fig. 1 (a) A solvent-controlled strategy for multicolour fluorescent CDs using citric acid and urea as precursors. (b) Photographs of the asprepared CDs dispersed in water (B-CDs), ethanol (G-CDs), formic acid (Y, R-CDs), under daylight (left) and UV irradiation (right). (c) Their normalized PL spectra. (d) The UV-vis absorption spectra of CDs. 


\section{Results and discussion}

We synthesized CDs solvothermally by heating a mixture of CA and urea in different solvents. As shown in Fig. 1(a), we used CA as the carbon source and urea as the nitrogen source. Multicolor CDs, i.e., B-, G-, Y-, and R-CDs, were successfully obtained by changing the solvent and mass ratio of the raw materials. Fig. 1(b) shows fluorescent images of the prepared CDs under daylight and $365 \mathrm{~nm}$ UV light. These solutions have different colors in daylight and bright blue, green, yellow, and red fluorescence can be seen under UV light (365 nm). Their PL emission maxima are located at 445, 513, 557, and $620 \mathrm{~nm}$, respectively (Fig. 1(c)). The UV-vis absorption spectra in Fig. 1(d) show that the four samples display similar absorption peaks in the high-energy absorption region (250-300 $\mathrm{nm}$ ). These are attributed to the $\pi-\pi^{*}$ transitions of conjugated $\mathrm{C}=\mathrm{C}$ and $\mathrm{C}=\mathrm{N}$ bonds. ${ }^{49}$ In the low-energy region, there is only one absorption peak for the B-CDs, G-CDs, and R-CDs, located at 336, 405, and $522 \mathrm{~nm}$, respectively. The Y-CDs give three absorption peaks, at 319,422 , and $539 \mathrm{~nm}$. These peaks arise from the $\mathrm{n}-\pi^{*}$ transitions of conjugated $\mathrm{C}=\mathrm{O}$ and $\mathrm{C}=\mathrm{N}$ bonds. The different absorptions of the four samples in the visible region imply different surface states. ${ }^{6,40,51}$

Our CDs show excitation independence, in agreement with previously reported results. ${ }^{49}$ The PL emission is independent of the excitation wavelength, within a certain range of excitation wavelengths (as shown in Fig. 2). The maximum excitation and emission wavelengths $\left(\lambda_{\mathrm{ex}} / \lambda_{\mathrm{em}}\right)$ of the B-CDs, G-CDs, Y-CDs, and R-CDs are 371/445 nm, 420/513 nm, 420/557 nm, and 522/ $620 \mathrm{~nm}$, respectively. There are overlaps between the maximum excitation wavelengths of the four CDs and the absorption peaks in the UV-vis spectra in the low-energy region; this indicates that the fluorescence is derived from the $n-\pi^{*}$ transitions of conjugated $\mathrm{C}=\mathrm{O}$ and $\mathrm{C}=\mathrm{N}$ structures. ${ }^{52}$ The time-resolved PL decay curve for each sample was also recorded. Fig. 3 shows that the data for all four samples can be fitted to dualexponential curves, with an average lifetime of 5.97, 10.98, 4.25, and 2.74 ns for the B-, G-, Y-, and R-CDs, respectively. The lifetime consists of two components, i.e., a short-lived component $\tau 1$ and a long-lived component $\tau 2$ [Table S1, (ESI) $\dagger$ ], which are ascribed to the recombination processes in the carbon core and the surface states, respectively. ${ }^{50}$ The absolute fluorescence QY at the maximum excitation wavelength of each sample was determined by using an integrating sphere; the values for the BCDs, G-CDs, Y-CDs, and R-CDs are 27.3\%, 31.1\%, 22.9\%, and $8.8 \%$, respectively (Table $\mathrm{S} 2$, ESI $\dagger$ ).

The morphologies and sizes of the CDs were investigated by TEM and AFM. Drops of dilute aqueous solutions of the CDs were deposited on carbon-coated copper grids for TEM and on silicon substrates for AFM. As shown in Fig. 4(a)-(d), all four samples consisted of homogeneous and well-dispersed nanoparticles of average sizes about $2.81 \mathrm{~nm}$ (B-CDs), $2.44 \mathrm{~nm}$ (GCDs), $2.57 \mathrm{~nm}$ (Y-CDs), and $4.27 \mathrm{~nm}$ (R-CDs). The HRTEM images in the insets show that the four samples were spherical, with visible crystalline lattice fringes. The interlayer spacing of $0.21 \mathrm{~nm}$ corresponds to the $d$-spacing of the graphene (100) planes. ${ }^{53}$ This shows that most of the CDs have uniform atomic arrangements, which indicates high degrees of crystallinity. The AFM images (Fig. 4(e-h)) show that the four samples mainly remained as individual particles and that only a small proportion of the CDs aggregated to larger particles. The particle heights of $c a .3 \mathrm{~nm}$ correspond to five to six layers of graphene. ${ }^{4}$
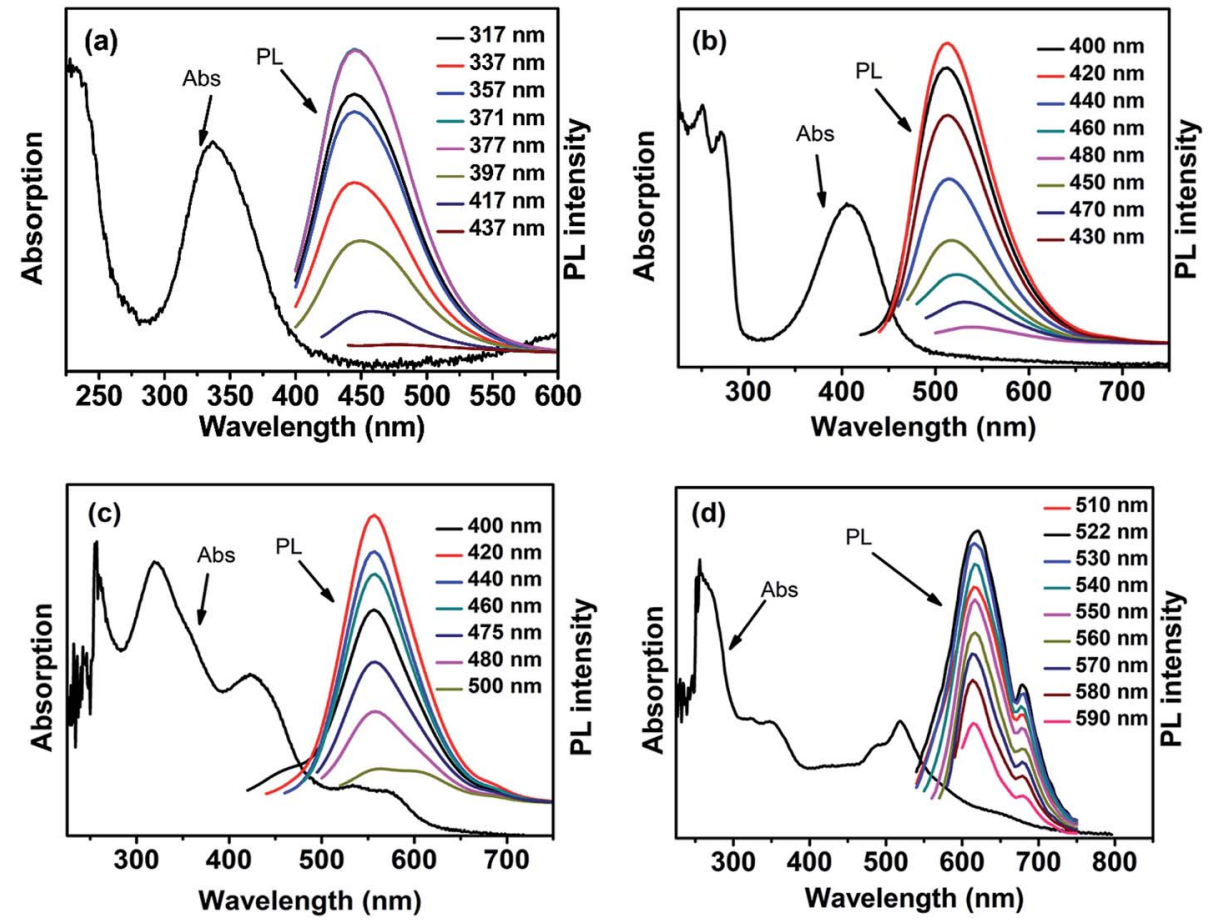

Fig. 2 Absorption spectra and PL emission spectra of (a) B-CDs, (b) G-CDs, (c) Y-CDs, and (d) R-CDs under excitation of different wavelengths of light (see the inset legends). 


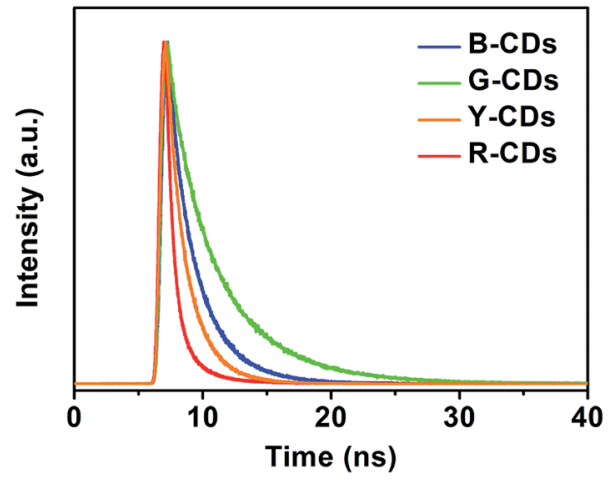

Fig. 3 Time-resolved PL decay curves of the four samples.

These results suggest that the nanoparticles might consist of nanocrystalline cores of graphitic $\mathrm{sp}^{2}$ carbon atoms..$^{54}$ The XRD patterns (Fig. S1, ESI $\dagger$ ) show a broad peak at about $26^{\circ}$; this corresponds to the (002) plane and is attributed to a graphitic structure with an interlayer spacing of $0.21 \mathrm{~nm}$. This is in agreement with the TEM and AFM results. ${ }^{52}$ The particle size has little influence on the maximum emission wavelength, in agreement with previously reported results. ${ }^{49}$

The Raman spectra provide strong evidence for ordered $\mathrm{sp}^{2}$ carbon and disordered $\mathrm{sp}^{3}$ carbon. Both peaks appear in the $1000-2000 \mathrm{~cm}^{-1}$ region; the $\mathrm{G}$ peak near $1590 \mathrm{~cm}^{-1}$ indicates $\mathrm{sp}^{2}$-hybridized carbon and the D peak at $1350 \mathrm{~cm}^{-1}$ represents a disordered or defective graphitized structure. ${ }^{40,49}$ Fig. 5 shows the Raman spectra of the four samples. Although the samples show identical Raman shifts, the $\mathrm{D}$ to $\mathrm{G}$ intensity ratios $\left(I_{\mathrm{D}} / I_{\mathrm{G}}\right)$ of the CDs vary, i.e., 0.80 for the $\mathrm{B}-, \mathrm{G}-$, and $\mathrm{Y}-\mathrm{CDs}$, and 1.06 for the $\mathrm{R}$-CDs. For the R-CDs, the D band is stronger than the $\mathrm{G}$ band, and these may be responsible for the redshift.

FTIR spectroscopy and XPS were performed to investigate the chemical bonds and functional groups in the prepared CDs. FTIR spectroscopy is a useful structure-sensitive technique for determining the bonding states of oxygen-containing groups on

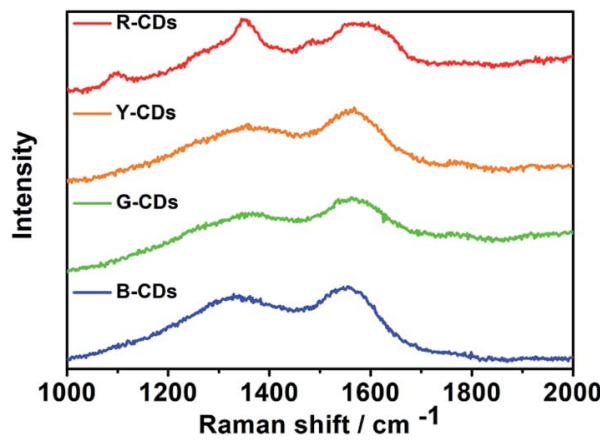

Fig. 5 Raman spectra of the four CDs.

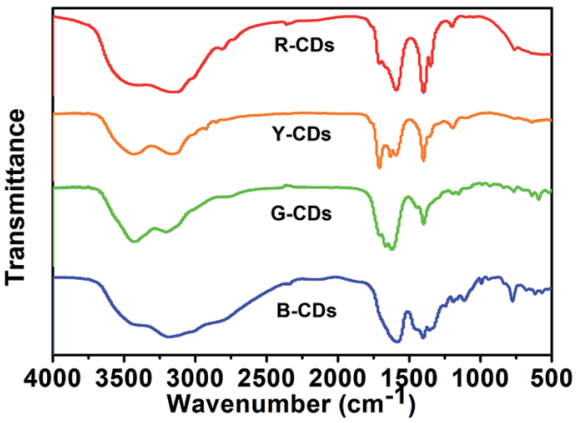

Fig. 6 FT-IR spectra of the four CDs.

the surfaces of samples. The FTIR spectra of the four samples (Fig. 6) show that all four samples have abundant polar functional groups, i.e., $\mathrm{O}-\mathrm{H}$ at $3434 \mathrm{~cm}^{-1}, \mathrm{~N}-\mathrm{H}$ at $3185 \mathrm{~cm}^{-1}, \mathrm{C}=\mathrm{O}$ at $1710 \mathrm{~cm}^{-1}$, and $\mathrm{C}-\mathrm{O}$ at $1200 \mathrm{~cm}^{-1}$; the samples therefore have excellent solubility in polar solvents. ${ }^{54}$ The stretching vibrations for $\mathrm{C}=\mathrm{C}$ at $1595 \mathrm{~cm}^{-1}$ and $\mathrm{C}-\mathrm{N}$ at $1400 \mathrm{~cm}^{-1}$ were observed for each sample, which indicates the formation of polyaromatic structures during CDs synthesis. ${ }^{42,54}$

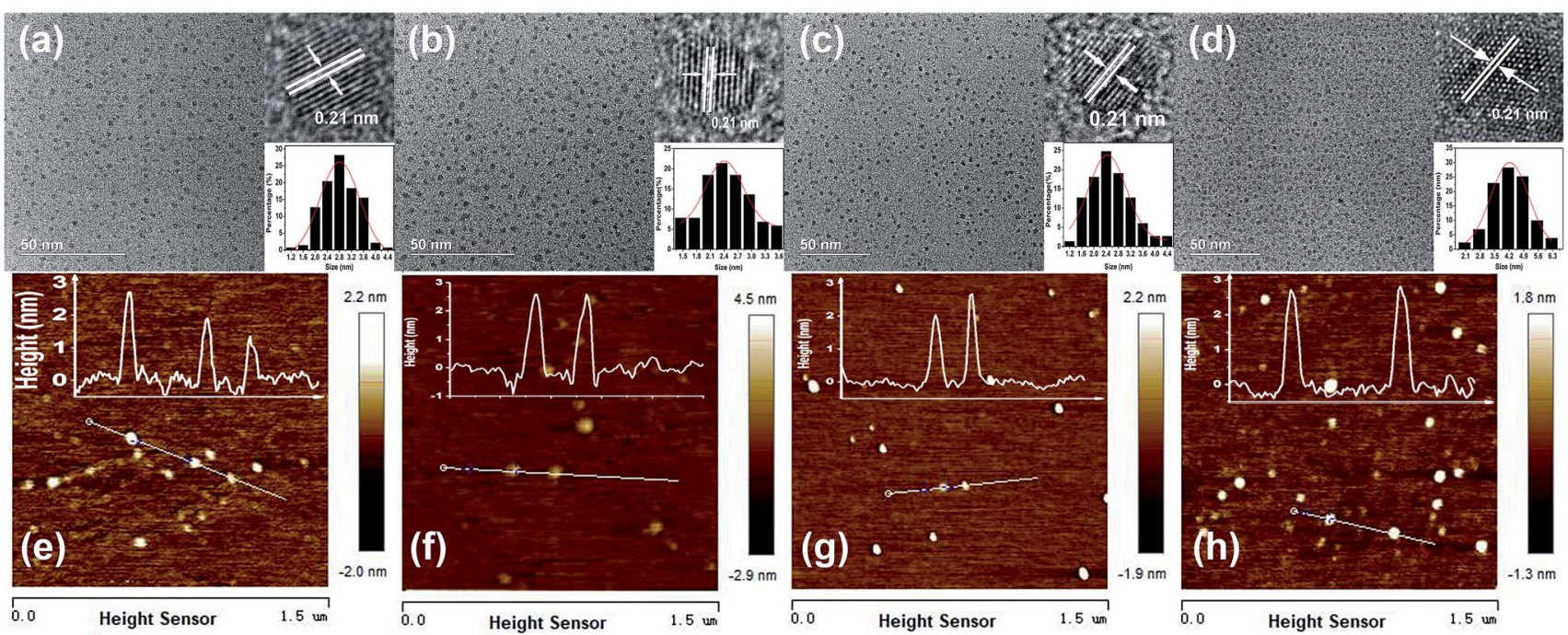

Fig. 4 TEM images of (a) B-CDs, (b) G-CDs, (c) Y-CDs, and (d) R-CDs. Insets are the corresponding high resolution TEM at top right corner and particle size distributions at bottom right corner. AFM images of (e) B-CDs, (f) G-CDs, (g) Y-CDs, and (h) R-CDs. 
A comparison of the FTIR spectra of the four samples shows that the intensities of the $\mathrm{C}-\mathrm{N}$ stretching vibrations at $1400 \mathrm{~cm}^{-1}$ increase from the B-CDs to R-CDs. This indicates a gradual increase in the amount of nitrogen-doped polyaromatic structures, accompanied by PL emission redshift of the CDs. ${ }^{50}$ XPS is a potent technique for determining the chemical structures and compositions of carbon materials because the binding energy of the core-level peak provides elemental and chemical information. The full survey XPS spectra of all four samples (Fig. S2, ESI $\dagger$ ) show three peaks, at 285,400 , and $531 \mathrm{eV}$, which correspond to $\mathrm{C} 1 \mathrm{~s}, \mathrm{~N} 1 \mathrm{~s}$, and $\mathrm{O} 1 \mathrm{~s}$, respectively. This indicates that the CDs, which were prepared from urea, are doped with nitrogen. The percentage of carbon increases, and the percentage of oxygen decreases, from the BCDs to R-CDs (Table S3, ESI $\dagger$ ). Fig. 7(a)-(d) show highresolution $\mathrm{C} 1 \mathrm{~s}, \mathrm{~N} 1 \mathrm{~s}$, and $\mathrm{O} 1 \mathrm{~s}$ XPS spectra of all four samples. The C 1s spectra can be fitted to three pronounced graphitic peaks, at 284.5, 286.6, and $288.3 \mathrm{eV}$, which correspond
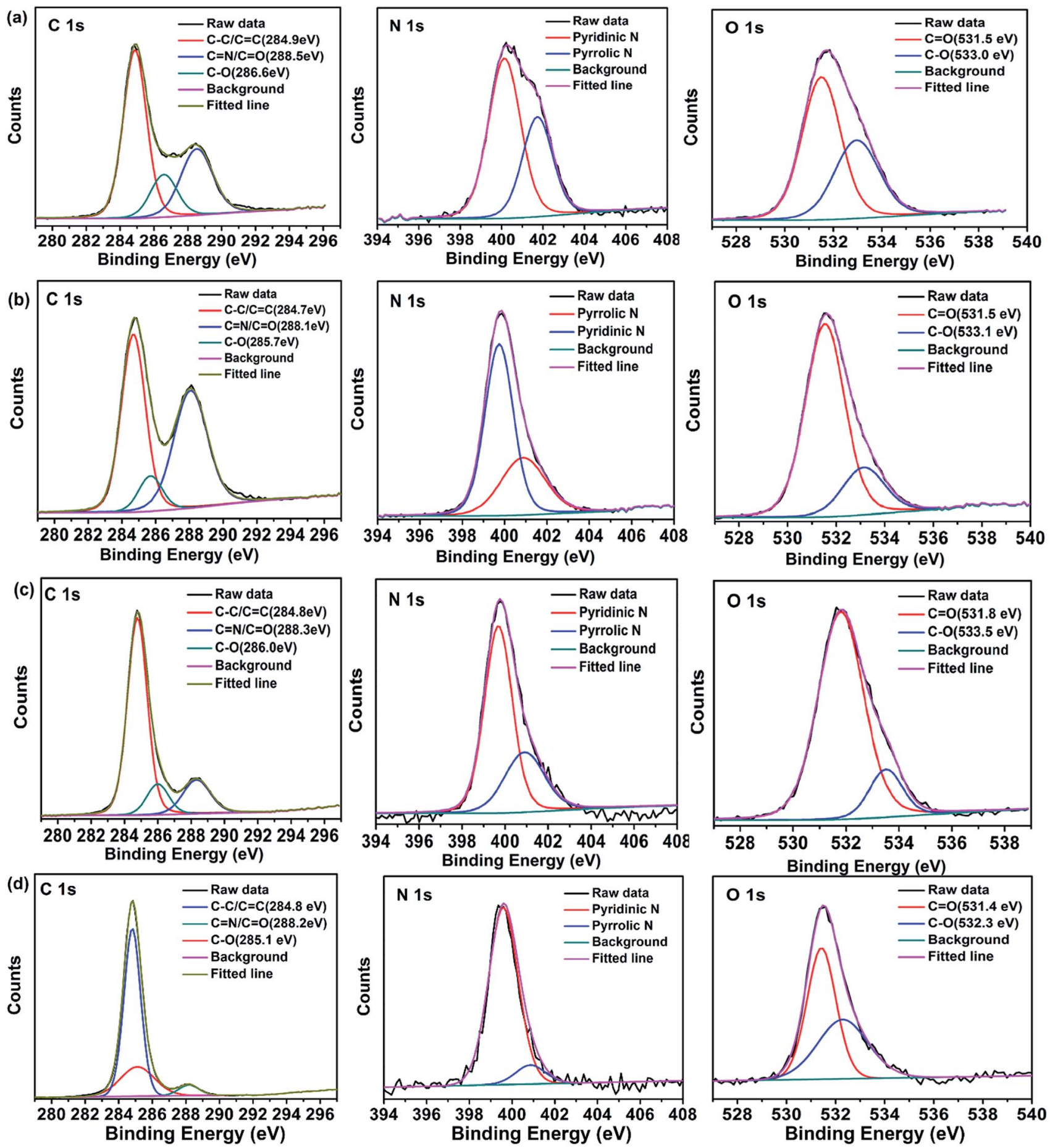

Fig. 7 High-resolution XPS C 1s, N 1s, O 1s spectra of (a) B-CDs, (b) G-CDs, (c) Y-CDs, and (d) R-CDs. 

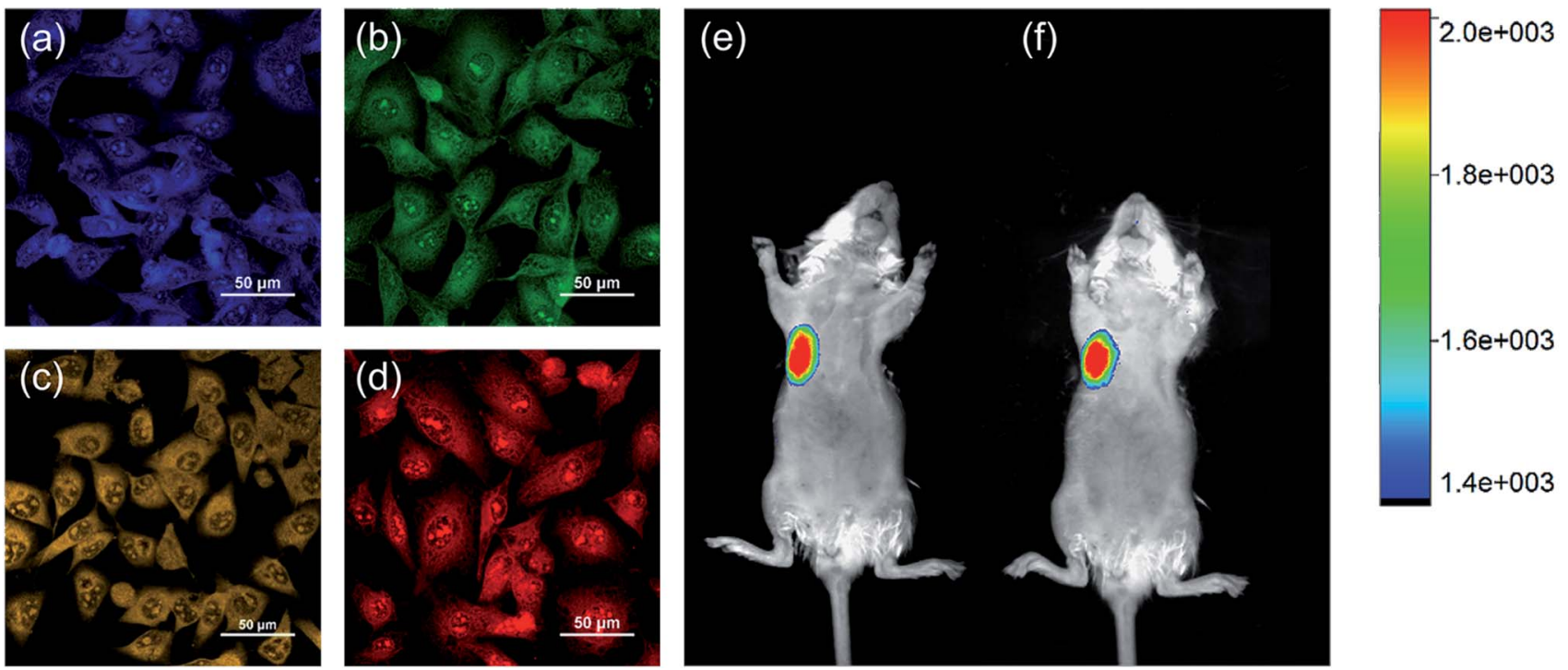

Fig. 8 Confocal fluorescence images of MDA-MB-231 cells incubated with $50 \mu \mathrm{g} \mathrm{mL} \mathrm{L}^{-1}$ of (a) B-CDs, (b) G-CDs, (c) Y-CDs, and (d) R-CDs for $24 \mathrm{~h}$. The four images were obtained under (a) $405 \mathrm{~nm}$, (b and c) $488 \mathrm{~nm}$ and (d) $561 \mathrm{~nm}$ excitation. In vivo fluorescence images of mice in injection site at (e) $0.5 \mathrm{~h}$ and (f) $1 \mathrm{~h}$ after subcutaneously injection of $500 \mu \mathrm{g} \mathrm{mL}{ }^{-1} \mathrm{R}-\mathrm{CDs}$. The colour bars represent the fluorescence intensity.

to $\mathrm{C}-\mathrm{C} / \mathrm{C}=\mathrm{C}, \mathrm{C}-\mathrm{O}$, and $\mathrm{C}=\mathrm{N} / \mathrm{C}=\mathrm{O}$, respectively. ${ }^{52}$ The $\mathrm{N} 1 \mathrm{~s}$ high-resolution XPS spectra show peaks for pyrrolic $\mathrm{N}(\mathrm{C}-\mathrm{N}-\mathrm{C})$ and pyridinic $\mathrm{N}^{42}$ The spectra clearly show that the pyridinic $\mathrm{N}$ content increases from B-, G-, Y-, to R-CDs; this may be responsible for the redshift of the PL emission (Table S4, ESI $\dagger$ ). The high-resolution O 1s XPS spectra contain two peaks, at 531 and $532 \mathrm{eV}$, which are associated with $\mathrm{C}=\mathrm{O}$ and $\mathrm{C}-\mathrm{O}$, respectively. ${ }^{49}$

The mechanism of the PL emissions of CDs has been widely studied, and the involvement of various processes and factors has been proposed, e.g., quantum size effects, carbon core states, surface states, and electron-hole recombination..$^{\mathbf{1 6 4 7 , 4 9 , 5 5}}$ The theoretical calculations performed by Wen et al. showed that the emission wavelength of graphene quantum dots depends strongly on the particle size. The PL emission wavelength redshifts with increasing size. This is because the energy gap decreases as a result of $\pi$-electron delocalization within the $\mathrm{sp}^{2}$ domain. ${ }^{56} \mathrm{Qu}$ et al. suggested that the PL emission redshift of carbon nanoparticles can be attributed to electron-hole recombination, i.e., the PL emission is an intrinsic state emission. ${ }^{53}$ Ding and coworkers hold the view that nitrogen- and oxygen-related surface states and nitrogen-derived structures in the carbon core are responsible for the red emission of R-CDs. ${ }^{40}$ The results of the present work show that there are differences among the surface states of the four samples and the pyridinic $\mathrm{N}$ content gradually increases from the $\mathrm{B}-\mathrm{CDs}$ to $\mathrm{R}$ CDs. Based on these and previously reported results, we believe that a combination of the surface states and nitrogenderived structures in the carbon cores accounts for the redshift of the PL emission.

The four prepared CDs show strong PL emissions at 445, 513, 557 , and $620 \mathrm{~nm}$ under excitation at 371, 420, 420, and $522 \mathrm{~nm}$, respectively. The CDs are therefore excellent probes for bioimaging; this was tested by imaging MDA-MB-231 cells. Before being used in biological applications, the PL stabilities of the four prepared CDs were investigated under different conditions. Fig. S3-S5 in the ESI $\uparrow$ show that the PL emissions are stable in water and high-ionic-strength solutions; the PL intensity increases with increasing of organic solvent. The cytotoxicity toward MDA-MB-231 cells was investigated by CCK-8 assays. The results (Fig. S6, ESI $\dagger$ ) show that over $70 \%$ of MDA-MB-231 cells were still alive after incubation with $50 \mu \mathrm{g} \mathrm{mL} \mathrm{m}^{-1}$ of each type of CDs for $24 \mathrm{~h}$. This indicates that the cytotoxicity of all four CDs are much lower than those of many other inorganic particles and the CDs have potential applications in bioimaging. ${ }^{57}$ In vitro imaging with the CDs was investigated by incubating MDA-MB-231 cells at a concentration of $50 \mu \mathrm{g} \mathrm{mL}{ }^{-1}$ in PRMI-1640 medium for $24 \mathrm{~h}$ at $37{ }^{\circ} \mathrm{C}$. Confocal microscopic images were excited with lasers at excitation wavelengths of 405 , 488,488 , and $561 \mathrm{~nm}$, and detected in the ranges 424-550, 504$650,514-650$, and 584-720 $\mathrm{nm}$, respectively. As shown in Fig. 8(a)-(d), all four types of CDs were internalized into cells and emitted bright blue, green, yellow, or red fluorescence. On the basis of these results, we performed in vivo imaging experiments on mice. We used a Bruker in vivo imaging system with $610 \mathrm{~nm}$ excitation light and a $700 \mathrm{~nm}$ emission filter. As shown in Fig. 8(e) and (f), fluorescence was clearly observed at the injection site of a mouse that was subcutaneously injected with $50 \mu \mathrm{L}$ of R-CDs $\left(500 \mu \mathrm{g} \mathrm{mL}^{-1}\right)$. The fluorescence intensity was retained even after $1 \mathrm{~h}$, which implies that the R-CDs give stable fluorescence. These results confirm that R-CDs can efficiently penetrate mouse skin. Moreover, the mouse was still healthy after the injection, which confirmed that our CDs are biocompatible.

\section{Conclusions}

Biocompatible, low-toxic, multicolor fluorescent probes were successfully synthesized by a solvothermal method from cheap, readily available, non-toxic materials, namely CA and urea. The 
probes emitted bright blue, green, yellow, and red fluorescence. All four prepared CDs showed excitation-independent PL emissions in a certain wavelength range. The maximum emission wavelength ranged from $445 \mathrm{~nm}$ for B-CDs to $620 \mathrm{~nm}$ for R$\mathrm{CDs}$, and therefore covered the entire visible region. The absolute QYs for the B-, G-, Y-, and R-CDs were 27.3\%, 31.1\%, 22.9\%, and $8.8 \%$, respectively. The differences among the fluorescence properties of these four CDs arise from differences among the surface states and contents of pyridinic N. An increase in the pyridinic $\mathrm{N}$ content can redshift the maximum emission wavelength. These four CDs were stable at high salt concentrations and showed low cytotoxicity. They were successfully used for imaging MDA-MB-231 cells and subcutaneous imaging in mice. The results of this research will be useful to others working in this and related fields.

\section{Conflicts of interest}

There are no conflicts to declare.

\section{Acknowledgements}

This work was supported by Shenzhen Strategic Emerging Industry Development Special funds (JCYJ20170816143646446), Shenzhen Science and Technology Research and Development funds (GJHZ20170314164935502).

\section{References}

1 X. Xu, R. Ray, Y. Gu, H. J. Ploehn, L. Gearheart, K. Raker and W. A. Scrivens, J. Am. Chem. Soc., 2004, 126, 12736-12737.

2 Y. Zhuo, H. Miao, D. Zhong, S. Zhu and X. Yang, Mater. Lett., 2015, 139, 197-200.

3 S. Zhu, Q. Meng, L. Wang, J. Zhang, Y. Song, H. Jin, K. Zhang, H. Sun, H. Wang and B. Yang, Angew. Chem., Int. Ed., 2013, 52, 3953-3957.

4 J. Tang, B. Kong, H. Wu, M. Xu, Y. Wang, Y. Wang, D. Zhao and G. Zheng, Adv. Mater., 2013, 25, 6569-6574.

5 M. Z. Fahmi, A. Haris, A. J. Permana, D. L. N. Wibowo, B. Purwanto, Y. L. Nikmah and A. Idris, Rsc Adv., 2018, 8, 38376-38383.

6 S. Sun, L. Zhang, K. Jiang, A. Wu and H. Lin, Chem. Mater., 2016, 28, 8659-8668.

7 Z. Zhang, Y. Pan, Y. Fang, L. Zhang, J. Chen and C. Yi, Nanoscale, 2016, 8, 500-507.

8 Z. Qian, X. Shan, L. Chai, J. Ma, J. Chen and H. Feng, ACS Appl. Mater. Interfaces, 2014, 6, 6797-6805.

9 A. Kumar, A. R. Chowdhuri, D. Laha, T. K. Mahto, P. Karmakar and S. K. Sahu, Sens. Actuators, B, 2017, 242, 679-686.

10 V. Raveendran, A. R. S. Babu and N. K. Renuka, Rsc Adv., 2019, 9, 12070-12077.

11 D. Qu, Z. Sun, M. Zheng, J. Li, Y. Zhang, G. Zhang, H. Zhao, X. Liu and Z. Xie, Adv. Opt. Mater., 2015, 3, 360-367.

12 T. Feng, Q. Zeng, S. Lu, X. Yan, J. Liu, S. Tao, M. Yang and B. Yang, ACS Photonics, 2017, 5, 502-510.
13 X. Guo, Y. Zhu, L. Zhou, L. Zhang, Y. You, H. Zhang and J. Hao, Rsc Adv., 2018, 8, 38091-38099.

14 Z. Qian, J. Ma, X. Shan, L. Shao, J. Zhou, J. Chen and H. Feng, Rsc Adv., 2013, 3, 14571.

15 S. Zhu, J. Shao, Y. Song, X. Zhao, J. Du, L. Wang, H. Wang, K. Zhang, J. Zhang and B. Yang, Nanoscale, 2015, 7, 79277933.

16 L. Bao, C. Liu, Z. L. Zhang and D. W. Pang, Adv. Mater., 2015, 27, 1663-1667.

17 L. Bao, Z. L. Zhang, Z. Q. Tian, L. Zhang, C. Liu, Y. Lin, B. Qi and D. W. Pang, Adv. Mater., 2011, 23, 5801-5806.

18 P. Anilkumar, X. Wang, L. Cao, S. Sahu, J. H. Liu, P. Wang, K. Korch, K. N. Tackett 2nd, A. Parenzan and Y. P. Sun, Nanoscale, 2011, 3, 2023-2027.

19 C. K. Chua, Z. Sofer, P. Simek, O. Jankovsky, K. Klimova, S. Bakardjieva, S. H. Kuckova and M. Pumera, ACS Nano, 2015, 9, 2548-2555.

20 X. Meng, Q. Chang, C. Xue, J. Yang and S. Hu, Chem. Commun., 2017, 53, 3074-3077.

21 H. Liu, T. Ye and C. Mao, Angew. Chem., Int. Ed. Engl., 2007, 46, 6473-6475.

22 D. Reyes, M. Camacho, M. Camacho, M. Mayorga, D. Weathers, G. Salamo, Z. Wang and A. Neogi, Nanoscale Res. Lett., 2016, 11, 424.

23 J. Zhou, C. Booker, R. Li, X. Zhou, T.-K. Sham, X. Sun and Z. Ding, J. Am. Chem. Soc., 2007, 129, 744-745.

24 R. R. Xiaoyou Xu, Y. Gu, H. J. Ploehn, L. Gearheart, K. Raker and W. A. Scrivens, J. Am. Chem. Soc., 2004, 126, 1273612737.

25 X. Tan, Y. Li, X. Li, S. Zhou, L. Fan and S. Yang, Chem. Commun., 2015, 51, 2544-2546.

26 B. Das, P. Dadhich, P. Pal, P. K. Srivas, K. Bankoti and S. Dhara, J. Mater. Chem. B, 2014, 2, 6839-6847.

27 L. Pan, S. Sun, A. Zhang, K. Jiang, L. Zhang, C. Dong, Q. Huang, A. Wu and H. Lin, Adv. Mater., 2015, 27, 77827787.

28 H. Huang, J.-J. Lv, D.-L. Zhou, N. Bao, Y. Xu, A.-J. Wang and J.-J. Feng, Rsc Adv., 2013, 3, 21691.

29 Q. Wang, X. Liu, L. Zhang and Y. Lv, Analyst, 2012, 137, 53925397.

30 R. R. Gaddam, D. Vasudevan, R. Narayan and K. V. S. N. Raju, RSC Adv., 2014, 4, 57137-57143.

31 D. Zhong, H. Miao, K. Yang and X. Yang, Mater. Lett., 2016, 166, 89-92.

32 C. Zhao, Y. Jiao, F. Hu and Y. Yang, Spectrochim. Acta, Part A, 2018, 190, 360-367.

33 V. N. Mehta, S. Jha, H. Basu, R. K. Singhal and S. K. Kailasa, Sens. Actuators, B, 2015, 213, 434-443.

34 V. N. Mehta, S. Jha and S. K. Kailasa, Mater. Sci. Eng., C, 2014, 38, 20-27.

35 Y. Liu, N. Xiao, N. Gong, H. Wang, X. Shi, W. Gu and L. Ye, Carbon, 2014, 68, 258-264.

36 S. Sahu, B. Behera, T. K. Maiti and S. Mohapatra, Chem. Commun., 2012, 48, 8835.

37 L. Li, R. Zhang, C. Lu, J. Sun, L. Wang, B. Qu, T. Li, Y. Liu and S. Li, J. Mater. Chem. B, 2017, 5, 7328-7334. 
38 J. Ge, Q. Jia, W. Liu, L. Guo, Q. Liu, M. Lan, H. Zhang, X. Meng and P. Wang, Adv. Mater., 2015, 27, 4169-4177.

39 S. Lu, L. Sui, J. Liu, S. Zhu, A. Chen, M. Jin and B. Yang, Adv. Mater., 2017, 29, 1603443.

40 H. Ding, J. S. Wei, N. Zhong, Q. Y. Gao and H. M. Xiong, Langmuir, 2017, 33, 12635-12642.

41 S. Qu, D. Zhou, D. Li, W. Ji, P. Jing, D. Han, L. Liu, H. Zeng and D. Shen, Adv. Mater., 2016, 28, 3516-3521.

42 K. Jiang, S. Sun, L. Zhang, Y. Wang, C. Cai and H. Lin, ACS Appl. Mater. Interfaces, 2015, 7, 23231-23238.

43 J. Chen, J. Liu, J. Li, L. Xu and Y. Qiao, J. Colloid Interface Sci., 2017, 485, 167-174.

44 C. Wang, Z. Xu, H. Cheng, H. Lin, M. G. Humphrey and C. Zhang, Carbon, 2015, 82, 87-95.

45 S. Chandra, T. K. Mahto, A. R. Chowdhuri, B. Das and S. k. Sahu, Sens. Actuators, B, 2017, 245, 835-844.

46 Y. Song, S. Zhu, S. Xiang, X. Zhao, J. Zhang, H. Zhang, Y. Fu and B. Yang, Nanoscale, 2014, 6, 4676-4682.

47 K. Jiang, S. Sun, L. Zhang, Y. Lu, A. Wu, C. Cai and H. Lin, Angew. Chem., Int. Ed. Engl., 2015, 54, 5360-5363.
48 Y. Mu, N. Wang, Z. Sun, J. Wang, J. Li and J. Yu, Chem. Sci., 2016, 7, 3564-3568.

49 H. Ding, S. B. Yu, J. S. Wei and H. M. Xiong, ACS Nano, 2016, 10, 484-491.

50 H. Ding, J. S. Wei, P. Zhang, Z. Y. Zhou, Q. Y. Gao and H. M. Xiong, Small, 2018, e1800612.

51 J. Zhan, B. Geng, K. Wu, G. Xu, L. Wang, R. Guo, B. Lei, F. Zheng, D. Pan and M. Wu, Carbon, 2018, 130, 153-163.

52 X. Miao, D. Qu, D. Yang, B. Nie, Y. Zhao, H. Fan and Z. J. A. M. Sun, Adv. Mater., 2017, 30, 1870002.

53 S. Qu, X. Liu, X. Guo, M. Chu, L. Zhang and D. Shen, Adv. Funct. Mater., 2014, 24, 2689-2695.

54 H. Wang, C. Sun, X. Chen, Y. Zhang, V. L. Colvin, Q. Rice, J. Seo, S. Feng, S. Wang and W. W. Yu, Nanoscale, 2017, 9, 1909-1915.

55 L. Guo, J. Ge, W. Liu, G. Niu, Q. Jia, H. Wang and P. Wang, Nanoscale, 2016, 8, 729-734.

56 X. Wen, P. Yu, Y.-R. Toh, X. Hao and J. Tang, Adv. Opt. Mater., 2013, 1, 173-178.

57 J. Kim, J. Park, H. Kim, K. Singha and W. J. Kim, Biomaterials, 2013, 34, 7168-7180. 